\title{
Initial state geometry and fluctuations in deformed and asymmetric nuclear collisions in the IP-Glasma framework
}

\author{
Björn Schenke ${ }^{a}$, Prithwish Tribedy ${ }^{\mathrm{b}}$, Raju Venugopalan ${ }^{\mathrm{a}}$ \\ ${ }^{a}$ Physics Department, Brookhaven National Laboratory, Upton, NY 11973, USA \\ ${ }^{b}$ Variable Energy Cyclotron Centre, 1/AF Bidhan Nagar, Kolkata 700064, India
}

\begin{abstract}
The IP-Glasma model of initial conditions based on the ab initio color glass condensate framework successfully explains most of the bulk features of the global data for various systems like $\mathrm{p}+\mathrm{p}, \mathrm{p}+\mathrm{A}$ and $\mathrm{A}+\mathrm{A}$ over a wide range of energies. We employ this framework to study deformed $\mathrm{U}+\mathrm{U}$ collisions, asymmetric $\mathrm{Cu}+\mathrm{Au}$ collisions and the effect of deformation in $\mathrm{Au}+\mathrm{Au}$ collisions at RHIC. A combined study of these heavy ion systems with varying initial geometries can provide a unique opportunity to determine the origin of different sources of fluctuations that affect global observables like multiplicity and flow. We study the sensitivity of multiplicity, eccentricity and their event-by-event distributions to the details of initial state geometry. Results are compared to a two component MC-Glauber model implementation that includes Negative-Binomial multiplicity fluctuations. We argue that the measurements of global observables for these systems at RHIC can constrain the mechanism of multi particle production.
\end{abstract}

Keywords:

heavy ion collisions, deformed nuclei, particle production

\section{Introduction}

Event by event fluctuations of global observables like multiplicity and flow are important observables to constrain the initial conditions for heavy ion collisions. Such fluctuations are sensitive to the details of the initial state geometry and the mechanism of multi particle production. Multiple sources contribute to the initial state fluctuations. They include geometric fluctuations due to variation of the impact parameter and the Euler angles associated with the orientations of the colliding nuclei, as well as quantum fluctuations at the nucleon scale. The fluctuations generate a lumpy distribution of color charges inside the colliding nuclei. For each configuration of the color charge distribution there are additional sub-nucleonic fluctuations, the dynamics of which is governed by the saturated nuclear wave function at small Bjorken $x[1,2]$. The combined effect of these fluctuations leads to the fluctuations of initial gluon distributions and therefore the fluctuations of the global observables.

In principle it is difficult to disentangle the role of individual sources of initial state fluctuations and the mechanism of particle production from the experimental observables. Studies of systems with varying initial geometry can provide a unique opportunity in this context. In collisions of deformed nuclei certain configurations of collision geometries can be triggered on, and the role of individual initial state fluctuations and the mechanism of particle production can be studied [3].

In Ref. [3] we have employed the IP-Glasma model and the two component MC-Glauber models of initial conditions to study the global observables for asymmetric $\mathrm{Cu}+\mathrm{Au}$ collisions, unpolarized deformed $\mathrm{U}+\mathrm{U}$ collisions (Random $\mathrm{U}+\mathrm{U}$ ) and the effect of moderate deformation in $\mathrm{Au}+\mathrm{Au}$ collisions. The STAR collaboration has recently investigated $\mathrm{U}+\mathrm{U}$ and $\mathrm{Au}+\mathrm{Au}$ collisions at RHIC by triggering the ultra central events using the number of spectator 


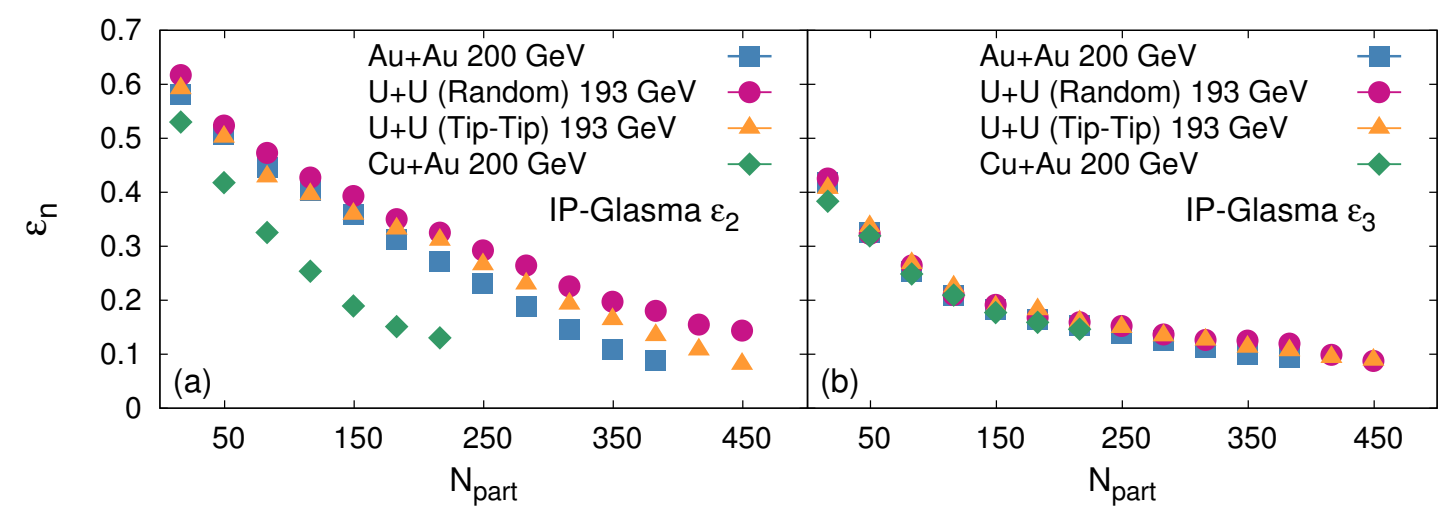

Figure 1. (color online) Centrality dependence of the initial ellipticity (a) and triangularity (b) for different colliding systems in the IP-Glasma model. Figure (a) is taken from Ref. [3]

neutrons [4]. Since the IP-Glasma model and the two component MC-Glauber model include qualitatively different mechanisms of particle production, a direct comparison of our predictions to the STAR data can constrain the model of initial conditions at RHIC.

\section{Models of particle production}

The IP-Glasma model, based on the framework of Color Glass Condensate (CGC) that incorporates different sources of initial state fluctuations, provides an ab initio mechanism of multi particle production [1, 2]. The IPGlasma model combines the IP-Sat model of HERA DIS [5] and the Yang-Mills dynamics of classical Glasma gluon fields [6]. The model naturally produces Negative-Binomial multiplicity fluctuations and (when combined with fluid dynamic simulations) flow harmonics compatible with experimental data for a wide range of systems and collision energies $[7,8]$.

Another widely used model of multi particle production for heavy ion collisions is the two component model [9]. The input to this model is the collision geometry from the MC-Glauber model. The model then assigns a specific weight for particle production to the participants $\left(N_{\text {part }}\right)$ and the binary collisions $\left(N_{\text {coll }}\right)$. For a quantitative comparison to IP-Glasma results we tune the parameters of the MC-Glauber model to fit the min-bias multiplicity distribution in the IP-Glasma model for $\mathrm{Au}+\mathrm{Au}$ collisions at $200 \mathrm{GeV}$.

The qualitative differences between the mechanism of particle production in the two models are as follows. In the CGC picture multiplicity is proportional to the quantity $\left\langle Q_{\mathrm{S}}^{2} S_{\perp}\right\rangle / \alpha_{S}\left(Q_{\mathrm{S}}^{2}\right)$, where $Q_{\mathrm{S}}$ is the smaller one of the intrinsic saturation scales of the two colliding nuclei. The saturation scale of each colliding nuclei is proportional to its color charge density which depends on the average numbers of nucleons lined up along the direction of collision, therefore sensitive to the shape of the nuclei. However, it turns out that the combination $\left\langle Q_{S}^{2} S_{\perp}\right\rangle$ is insensitive to the nuclear shape, since configuration of larger $Q_{S}$ are usually compensated by small transverse area $S_{\perp}$. Therefore only a logarithmic dependence of multiplicity on collision geometry is introduced through $\alpha_{S}$ in this picture. On the other hand, in the two component model picture the multiplicity is assumed to be proportional partly to $N_{\text {part }}$ and partly to $N_{\text {coll }}$. This assumption introduces a strong dependence of the multiplicity on the collision geometry, since $N_{\text {coll }}$ is strongly dependent on how many nucleons line up along the collision direction. Therefore a stronger correlation is expected in the two-component model as compared to the CGC picture.

\section{Eccentricity and its fluctuations}

The event averaged eccentricities are sensitive to the different length scales of transverse density fluctuations near the surface of the system. In Fig.1 we present results for the centrality dependence of the event averaged ellipticity $\left(\varepsilon_{2}\right)$ and triangularity $\left(\varepsilon_{3}\right)$ for different collision systems such as $\mathrm{Au}+\mathrm{Au}, \mathrm{Cu}+\mathrm{Au}$, random $\mathrm{U}+\mathrm{U}$, and tip-tip $\mathrm{U}+\mathrm{U}$ 


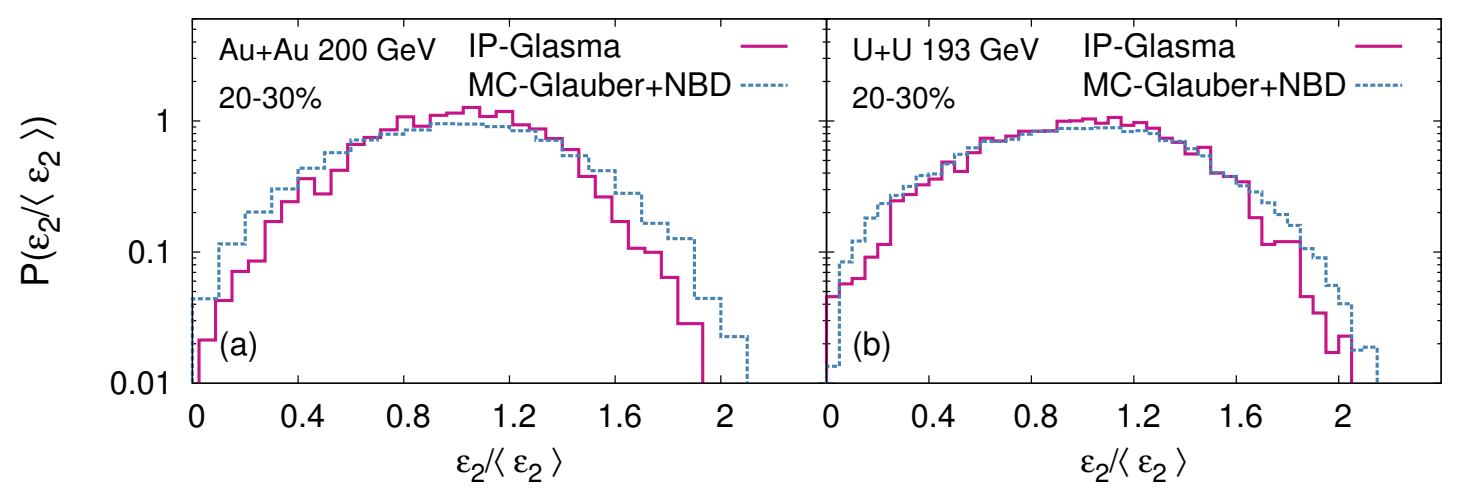

Figure 2. Probability distribution of the scaled ellipticity for 20-30\% Au+Au and U+U collisions from Ref. [3]

collisions at RHIC. For the latter the major axes of the two colliding nuclei line up along the direction of collision. It turns out that ellipticity (Fig.1(a)) is very sensitive to the initial geometry and centrality of the collisions. However, triangularity (Fig.1(b)) and other fluctuation driven moments are insensitive to the colliding systems.

The scaled probability distribution of eccentricities provides very good estimation of the flow harmonic distributions for central and mid-central collisions [7]. The distribution of $v_{n}$ did a very good job in constraining different models of initial conditions at LHC $[10,7]$. Such probability distributions contain information about cumulants of $\varepsilon_{n}$ or $v_{n}$ to all orders. In Fig. 2, we present results for the scaled ellipticity distributions from the IP-Glasma and the twocomponent MC-Glauber model for 20-30\% Au+Au and U+U collisions. The centrality selection for both the models is done using the corresponding min-bias multiplicity distribution, introducing an indirect dependence on the latter. For both systems, the MC-Glauber model predicts larger widths as compared to the IP-Glasma model. Experimental measurements of $v_{n}$ distributions at RHIC can therefore constrain the models of initial conditions.

\section{Correlation between multiplicity and eccentricity}

The correlation between event-by-event multiplicity and eccentricity is an important observable to constrain the mechanism of multi particle production. Triggering ultra central full overlap events for deformed nuclear collisions, one can constrain the initial geometry to fluctuate between two limiting geometries which are either tip-tip or sideside. For $\mathrm{U}+\mathrm{U}$ collisions that have prolate deformation, tip-tip configurations correspond to smaller $\varepsilon_{2}$ and larger number of binary collisions. The side-side configurations for $\mathrm{U}+\mathrm{U}$ collisions correspond to larger $\varepsilon_{2}$ and smaller number of binary collisions. For $\mathrm{Au}+\mathrm{Au}$ collisions this is the other way around when assuming an oblate deformation of the gold nucleus. As a result the two component MC-Glauber model predicts a strong anti-correlation for $\mathrm{U}+\mathrm{U}$ collisions and a strong correlation for $\mathrm{Au}+\mathrm{Au}$ collisions between $\varepsilon_{2}$ and multiplicity. In the CGC picture no such strong (anti-) correlation is expected.

In Fig. 3 we show the variations of $\varepsilon_{2}$ as a function of scaled multiplicity for $0-0.1 \%$ and $0-1 \%$ central collisions. These events are selected by applying cuts on the number of spectator neutrons similar to the STAR collaboration [4]. For U+U collisions, the IP-Glasma model predicts a weaker anti-correlation as compared to the MC-Glauber model. For $\mathrm{Au}+\mathrm{Au}$ collisions, the IP-Glasma predicts a qualitatively different trend as compared to the MC-Glauber model. For $0-1 \% \mathrm{Au}+\mathrm{Au}$ collisions, a positive slope for the correlation between $\varepsilon_{2}$ and multiplicity is seen in the MCGlauber model, whereas IP-Glasma predicts a small negative slope. Preliminary results on the $v_{2}$ of charged particles as a function of the scaled multiplicity have been compared to both IP-Glasma and MC-Glauber model calculations by the STAR collaboration in Ref. [11]. The IP-Glasma model results were found to be more compatible with the experimental data. 


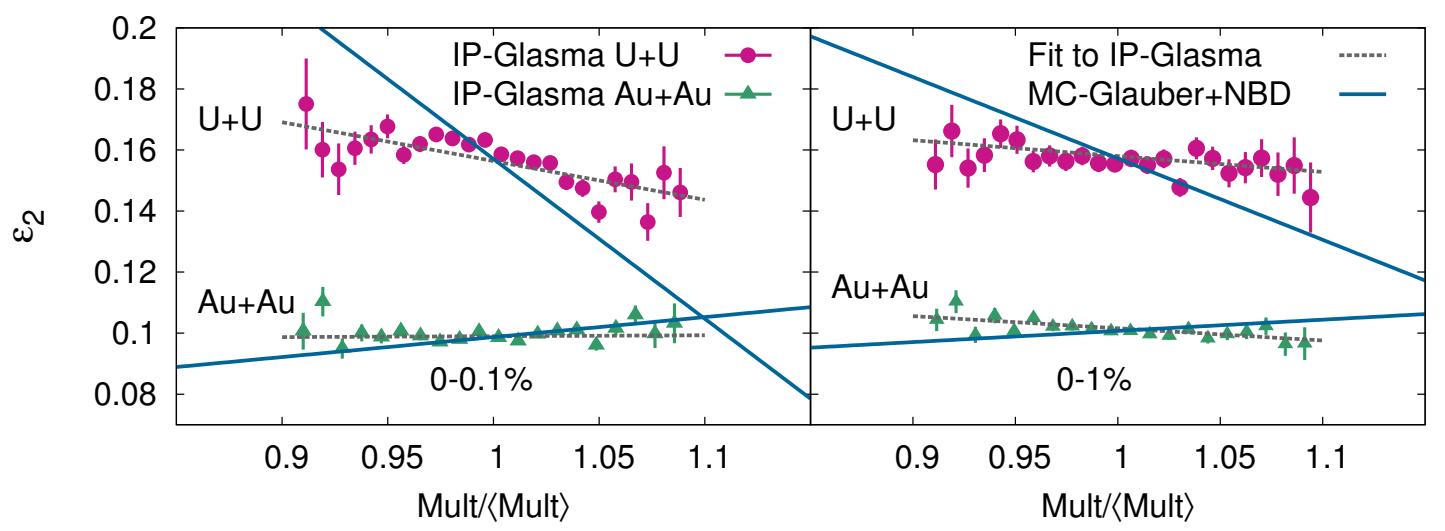

Figure 3. Correlations between ellipticity and scaled multiplicity for ultra central full-overlap events from Ref. [3]

\section{Conclusions}

We have studied the bulk observables for a wide range of collision geometries in the framework of the IP-Glasma model and two component MC-Glauber model. These two models include qualitatively different particle production mechanisms. For the IP-Glasma model, the event averaged ellipticities vary strongly with centrality and collision geometry. The triangularity for different systems follows a universal centrality dependence. For mid-central collisions the probability distribution of the ellipticity is found to be wider for the MC-Glauber model than the IP-Glasma model. These predictions can be directly compared to event by event distributions of scaled flow harmonics $v_{2} /\left\langle v_{2}\right\rangle$ to discriminate between the two models. An anti-correlation between ellipticity and scaled multiplicity was observed in ultra central $U+U$ collisions which is weaker for the IP-Glasma model as compared to the two component MCGlauber model. For Au+Au collisions a qualitatively different result was observed. Measurements of the correlation between $v_{2}$ and multiplicity by the STAR collaboration indicate that IP-Glasma results are consistent with the data.

\section{Acknowledgment}

This research used resources of NERSC, which is supported by the Office of Science of the US DOE under Contract No. DE-AC02-05CH11231, the DRONA and PRAFULLA cluster of Computer Division and the LHC grid computing centre at the Variable Energy Cyclotron Centre, supported by the Dept. of Atomic Energy, Government of India. BPS and RV are supported under DOE Contract No. DE-AC02-98CH10886.

\section{References}

[1] B. Schenke, P. Tribedy, R. Venugopalan, Fluctuating Glasma initial conditions and flow in heavy ion collisions, Phys. Rev. Lett. 108 (2012) 252301.

[2] B. Schenke, P. Tribedy, R. Venugopalan, Event-by-event gluon multiplicity, energy density and eccentricities at RHIC and LHC, Phys. Rev. C86 (2012) 034908.

[3] B. Schenke, P. Tribedy, R. Venugopalan, Initial state geometry and fluctuations in Au+Au, $\mathrm{Cu}+\mathrm{Au}$ and U+U collisions at RHIC, Phys. Rev. C 89 (2014) 064908. arXiv:1403.2232, doi:10.1103/PhysRevC.89.064908.

[4] H. Wang, P. Sorensen, Azimuthal anisotropy in U+U collisions at STAR arXiv:1406.7522.

[5] H. Kowalski, D. Teaney, An impact parameter dipole saturation model, Phys. Rev. D68 (2003) 114005. doi:10.1103/PhysRevD.68.114005.

[6] A. Krasnitz, R. Venugopalan, The initial energy density of gluons produced in very high energy nuclear collisions, Phys. Rev. Lett. 84 (2000) 4309-4312.

[7] C. Gale, S. Jeon, B. Schenke, P. Tribedy, R. Venugopalan, Event-by-event anisotropic flow in heavy-ion collisions from combined Yang-Mills and viscous fluid dynamics, Phys.Rev.Lett. 110 (2013) 012302. arXiv:1209.6330, doi:10.1103/PhysRevLett.110.012302.

[8] B. Schenke, P. Tribedy, R. Venugopalan, Multiplicity distributions in p+p, p+A and A+A collisions from Yang-Mills dynamics, Phys.Rev. C89 (2014) 024901. arXiv:1311.3636, doi:10.1103/PhysRevC.89.024901.

[9] D. Kharzeev, M. Nardi, Hadron production in nuclear collisions at RHIC and high density QCD, Phys.Lett. B507 (2001) 121-128.

[10] J. Jia, Measurement of the distributions of event-by-event flow harmonics in Pb-Pb Collisions at $\left.\sqrt{(} s_{n n}\right)=2.76$ TeV with the ATLAS detector, Nucl.Phys.A 904-905 2013 421c. arXiv:1209.4232.

[11] H. Wang, these proceedings. 